\title{
EVALUACIÓN DE PARÁMETROS BIOQUIMICOS Y MORFOGENETICOS EN LA SIMBIOSIS Azolla filiculoides - Anabaena azollae COMO RESPUESTA A LA INTERACCIÓN DE LA CALIDAD DE LUZ Y DOS NIVELES DE NITRÓGENO
}

Javier Mosquera Lenti ${ }^{1}$ y Abelardo Calderón Rodriguez ${ }^{2}$

\begin{abstract}
Resumen
En el presente trabajo experimental se evaluó el efecto producido por la interacción de tres calidades de luz (roja, blanca y azul) con dos niveles de nitrógeno (IRRI $+\mathrm{N}$ e IRRI -N) en la simbiosis Azolla filiculoides - Anabaena azollae, mediante el análisis de diversos parámetros como: la tasa de crecimiento, el contenido de clorofila, proteína y prolina, la actividad de las enzimas nitrato reductasa y nitrogenasa, así como la cantidad de células vegetativas y heterocistos presentes en cada tratamiento. Los resultados indicaron que las plantas crecidas en el medio sin nitrógeno presentaron una mayor tasa de crecimiento, así como una mayor cantidad del endosimbionte Anabaena azollae Strass. con relación a las plantas crecidas en el medio con nitrógeno, independientemente de la cantidad de luz. A su vez, las plantas crecidas en el medio IRRI con nitrógeno (IRRI $+\mathrm{N}$ ) presentaron un mayor contenido de clorofila así como de prolina con relación a las plantas crecidas en el medio IRRI sin nitrógeno (IRRI -N). En cuanto a la cantidad de proteína, las plantas iluminadas con luz azul, mostraron contenidos significativamente mayores que aquellas crecidas bajo luz blanca y roja.
\end{abstract}

Palabras clave: Azolla, Anabaena, Simbionte, Fijación de Nitrógeno, prolina.

\begin{abstract}
The objective of the present study was to investigate the interaction between three light qualities (red, white and blue) and two nitrogen levels (IRRI +N and IRRI -N) on the simbiosis Azolla filiculoides - Anabaena azollae through the evaluation of parameters such as: Growth rate of vegetative cells and heterocists, chlorophyll, protein and proline contents and the nitrogenase and nitrate reductase activities. The results show that the plants grown on IRRI -N medium had the greater rate of growth, and the best development of the endosimbiont Anabaena azollae Strass with relation the ones grown on IRRI $+\mathrm{N}$ medium, in all the light levels. On the other hand, the plants grown on IRRI $+\mathrm{N}$ medium show the greater content of chlorophylls, and proline in contrast with the plants grown on IRRI -N medium. With respect to the protein content, we found that the blue light has a positive effect, than those grown under white and red light, elevating the concentration in the plants Nitrogenase and nitrate reductase activities.
\end{abstract}

Keywords: Azolla, Anabaena, Simbiont, Nitrogen Fixation, proline.

\section{Introducción}

La Azolla filiculoides Lam (Figura 1) es un pequeño helecho acuático flotante que vive en muchos espejos de agua dulce y en áreas tropicales y es capaz de fijar nitrógeno atmosférico debido a la simbiosis que mantiene con la cianobacteria Anabaena azollae Stras (Figura 2) que habita en unas grandes cavidades en el lóbulo superior de la fronda de Azolla.

La fijación del nitrógeno se debe a la actividad de la enzima nitrogenasa en la cianobacteria. Esta enzima se encuentra presente en células especializadas denominados heterocistos, los cuales convierten en Nitrógeno molecular del aire en amoniaco, que posteriormente es incorporado en los compuestos nitrogenados tanto por la planta como por la cianobacteria. El simbionte de la cavidad foliar tiene de 15 a 20 por ciento de heterocistos comparado con las especies de Anabaena de vida libre que solo tiene un 5 por ciento de ellos.

En cuanto al hospedero Azolla, este contiene los pigmentos fotosintéticos clorofila $a$, clorofila $b$ y carotenoides asociados a los cloroplastos; mientras que los filamentos de Anabaena presentan clorofila a, ficobiliproteínas y carotenoides. Esta suma de pigmentos fotosintéticos principales y accesorios permite al organismo simbiótico ampliar el rango de utilización de la energía luminosa aprovechable. Es probable que esta característica sea la responsable del crecimiento rápido y la gran acumulación de biomasa en períodos cortos.

\footnotetext{
${ }^{1}$ Biólogo, asistente de Investigación del Laboratorio de Fisiología Vegetal- UNALM.

${ }^{2}$ Biólogo, M. Sc. Profesor Principal de Fisiología Vegetal Departamento de Biología UNALM.
} 
La Azolla, puede duplicarse aproximadamente cada 5 días bajo condiciones favorables.

Por otro lado, el incremento desmedido de la población mundial y la escasez mas frecuente de combustibles fósiles para fabricar fertilizantes nitrogenados hacen necesario voltear nuestra mirada hacia estas alternativas biológicas de bajo costo y nada contaminantes. La Azolla es el abono verde que más eficientemente se usa en el cultivo del arroz y puede ser trasladado aún a otros cultivos y por ello es necesario investigar varios aspectos en los strains disponibles en nuestro país.

\section{Revisión de literatura}

Las simbiosis entre cianobacterias y plantas ocurre en un amplio segmento del reino Plantae, incluyendo algunas Briofitas (como Anthoceros, Blasia, Cavicularia), una Pteridofita (Azolla), Gimnospermas (9 géneros de Cycas, Zamia, Macrozamia y Encefalartos) y una Angiosperma (Gunnera de la familia Halagoraceae) (Peters \& Calvert, 1983; Reddy \& Fisher, 1988 )

Todas las especies conocidas de Azolla contienen a la cianobacteria Anabaena azollae Strass; y están clasificadas sobre la base de sus estructuras reproductivas y vegetativas (Alves, 1985; Becking 1978; Fay, 1973; Neidhardt et al., 1990)

Este helecho acuático, es considerado actualmente como un abono verde entre otros países como Filipinas, China, India, Vietnam, Tailandia e Indonesia (Ali \& Malik, 1983; Alves, 1985; Peters \& Calvert, 1983; Tovar, 1993); sin embargo en nuestro país poco o nada se ha hecho para incorporarla en las prácticas agrícolas, mas aún tomando en cuenta el creciente costo de los fertilizantes nitrogenados y la necesidad de aplicar una agricultura orgánica.

La asociación Azolla-Anabaena es literalmente una fábrica viva flotante de compuestos nitrogenados, que usa la energía de la fotosíntesis para fijar el nitrógeno atmosférico. La asociación puede fijar entre 100 a 150 $\mathrm{Kg}$. de N2 atmosférico por hectárea por año en aproximadamente 40-60 toneladas de biomasa. La fijación del nitrógeno ocurre tanto de noche como de día, pero la tasa es más baja en la oscuridad. Sobre la base del peso seco contiene aproximadamente $23.8 \%$ de proteína cruda, 4.4\% de grasas 6,4\% de almidón y 9.5\% de fibra (Becking, 1978; Peters \& Calvert, 1983; Reddy \& Ficsher, 1988).

La presencia de nitrógeno como fertilizante, en cualquier calidad de luz o la interacción entre ambos deprime el crecimiento de Azolla y ello nos lleva a pensar que esta debe ser una condición estresante para Anabaena, por ello que utilizamos a la prolina como un indicador de stress. La síntesis de proteínas tanto estructurales como enzimáticas (Bates, 1973; Katiyar, 1996, Moses \& Chua, 1988; Yatazawa et al., 1980) y el número de células vegetativas y heterocistos también se verán afectados en cierta medida si las condiciones no son las óptimas como ocurre en otras especies (Calderón, 1988; Franco et al., 1979, Grossman et al., 1993; La Rue \& Kuez, 1973; Zavaleta, 1992).

\section{Materiales y métodos}

El presente estudio se realizó en el laboratorio de Fisiología Vegetal de la Universidad Nacional Agraria La Molina. Las plantas de Azolla fueron recolectadas en los pantanos de Villa y se aclimataron y multiplicaron vegetativamente durante un mes en agua fangosa.

Luego de pasar por este período de adaptación fueron trasladados a los medios de cultivos IRRI $+\mathrm{N}$ e IRRI -N (Tabla 1), en contenedores plásticos de 500 $\mathrm{ml}$ cada uno y a su vez, cada nivel de nitrógeno fue colocado bajo las diferentes calidades de luz (roja, azul y blanca) (Figura 3). El fotoperíodo fue de 12 horas de luz y 12 de oscuridad a temperaturas entre $23^{\circ} \mathrm{C}$ y $25^{\circ} \mathrm{C}$.

TABLA 1. (International Rice Research Institute)

\begin{tabular}{|l|c|l|c|}
\hline \multicolumn{2}{|c|}{ Medio IRRI +N } & \multicolumn{2}{c|}{ Medio IRRI -N } \\
\hline Macronutrientes & $(\mathrm{g} /$ litro $)$ & Macronutrientes & $(\mathrm{g} /$ litro $)$ \\
\hline $\mathrm{NH}_{4} \mathrm{NO}_{3}$ & 1.650 & $\cdots$ & $\ldots$ \\
$\mathrm{CaCl}_{2}$ & 0.333 & $\mathrm{CaCl}_{2}$ & 0.333 \\
$\mathrm{MgSO}_{4} 6 \mathrm{H}_{2} \mathrm{O}$ & 0.492 & $\mathrm{MgSO}_{4} 6 \mathrm{H}_{2} \mathrm{O}$ & 0.492 \\
$\mathrm{~K}_{2} \mathrm{SO}_{4}$ & 0.274 & $\mathrm{~K}_{2} \mathrm{SO}_{4}$ & 0.274 \\
$\mathrm{NaH}_{2} \mathrm{PO}_{4}$ & 0.120 & $\mathrm{NaH}_{2} \mathrm{PO}_{4}$ & 0.120 \\
\hline $\mathrm{Micronutrientes}^{2}$ & $(\mathrm{mg} / \mathrm{litro})$ & $\mathrm{Micronutrientes}$ & $(\mathrm{mg} /$ litro $)$ \\
\hline $\mathrm{Fe}$ & 0.2 & $\mathrm{Fe}$ & 0.2 \\
$\mathrm{Mn}$ & 0.1 & $\mathrm{Mn}$ & 0.1 \\
$\mathrm{Zn}$ & 0.012 & $\mathrm{Zn}$ & 0.012 \\
$\mathrm{Cu}$ & 0.005 & $\mathrm{Cu}$ & 0.005 \\
$\mathrm{Mo}$ & 0.005 & $\mathrm{Mo}$ & 0.005 \\
$\mathrm{~B}$ & 0.635 & $\mathrm{~B}$ & 0.635 \\
\hline
\end{tabular}

Los parámetros evaluados para cada nivel de nitrógeno y calidad de luz fueron los siguientes:

Tasa de crecimiento en términos de peso fresco y de área foliar. Cantidad de células vegetativas y heterocistos, el cual se hizo por conteo de células al microscopio en un número determinado de campos visuales.

Se determinó el contenido de clorofila $\mathrm{a}, \mathrm{b}$ y total siguiendo el método de De Motts y Wintermans (Calderón, 1998).

Se determinó el contenido de proteínas totales mediante el método de Biuret (Zabaleta, 1992). Se 
evaluó la prolina siguiendo el método propuesto por Bates.

Se determinó la actividad de las enzimas Nitrato Reductasa y Nitrogenasa por el método de NeyraHageman y la Rue-Kurs respectivamente.

El diseño experimental se ajustó a un experimento factorial de 2 x 3 (3) dentro de un diseño completamente al azar (DCA). La bondad de los tratamientos se probó con un análisis de variancia y las comparaciones mediante pruebas de Duncan.

Finalmente para relacionar los diversos parámetros se empleó la correlación de variables.

\section{Resultados y discusión}

Los resultados corresponden a un promedio de dos evaluaciones en momentos diferentes y se muestran en la Tabla 2.

Estos resultados indicaron una mayor tasa de crecimiento (tanto en peso como en área foliar) en las plantas crecidas en el medio IRRI -N con un promedio de $0.092 \mathrm{dí}^{-1}$, mientras que aquellas crecidas en el medio IRRI +N mostraron un promedio de $0.027 \mathrm{día}^{-1}$. Esta diferencia de alrededor del $340.7 \%$, significó que mientras Azolla podía duplicar su masa en 7.54 días en el medio IRRI-N, en el medio IRRI $+\mathrm{N}$ requirió de 25.2 días.

A su vez, el contenido de células vegetativas y heterocistos fue mucho mayor en las plantas crecidas en el medio sin nitrógeno con un promedio de 29.16 mientras que aquellas crecidas en el medio IRRI $+\mathrm{N}$ tuvieron un promedio de 10.44. Estas diferencias podrían deberse al tiempo de contacto de la planta con los iones $\mathrm{NO}_{3}{ }^{-}$y $\mathrm{NH}_{4}{ }^{+}$que podrían haberla intoxicado al no poder esta obtener las cadenas carbonadas necesarias para su óptima conversión en proteínas u otros compuestos. Estos resultados concuerdan con las observaciones de varios autores como Talley y Grilli, en lo concerniente a la disminución del crecimiento en las cianobacterias simbióticas, como respuesta a la incorporación de fuentes nitrogenadas en el medio de cultivo, lo cual trae como consecuencia la síntesis de sustancias efectoras en la planta que actúan sobre la cianobacteria, impidiendo la síntesis de ciertas enzimas necesarias para la formación e incorporación de aminoácidos esenciales para su crecimiento. Los contenidos de clorofila $\mathrm{a}, \mathrm{b}$ y totales fueron mayores en las plantas crecidas en el medio IRRI $+\mathrm{N}$ con todas las calidades de luz con un promedio de $0.085 \mathrm{mg} / \mathrm{g}$ $\mathrm{PF}$ mientras que las plantas tratadas con el medio IRRI-N presentaron en promedio $0.049 \mathrm{mg} / \mathrm{g} \mathrm{PF}$. El nitrógeno es un elemento estructural de las clorofilas y al estar disponible en la forma inorgánica $\left(\mathrm{NO}_{3}{ }^{-}\right.$, $\mathrm{NH}_{4}{ }^{+}$) en el medio, puede pasar fácilmente a formar parte de las moléculas fotosintéticas, mientras que el Nitrógeno fijado por los simbiontes mayormente se ubica en moléculas orgánicas y un menor porcentaje se incorpora en las clorofilas de hospedero y simbionte (Fay, 1973; Peters \& Calvert, 1983). Con respecto al contenido de proteínas totales, las plantas crecidas bajo la luz azul tuvieron un promedio de ambos niveles de nitrógeno $45.47 \mathrm{mg} / \mathrm{g}$, mientras que las crecidas bajo el efecto de la luz blanca y la luz roja tuvieron promedios de $26.08 \mathrm{mg} / \mathrm{g}$ y $29.68 \mathrm{mg} / \mathrm{g}$ respectivamente. De esta observación podemos decir que los porcentajes de proteínas totales en Azolla crecida bajo luz azul en ambos niveles de nitrógeno con relación a los tratamientos de luz blanca y roja fueron mayores en un $74.34 \%$ y $53.20 \%$ respectivamente.

Estos resultados corroboran los datos de Alves (1985) en lo referente a la proteína total en condiciones de campo (luz natural) que está entre 23 y $26 \%$ evaluado por el método de Kjeldhal. Para las otras calidades de luz no existen datos comparativos, sin embargo de lo observado en otras plantas por varios autores, como Odum, Salisbury, Nichiporovitch citado por Calderón (1988) la producción de proteínas se estimula con la luz azul, sugiriéndose una activación de las enzimas ligadas a la flavina al absorberse la luz azul por medio de cafactores flavinicos de color amarillo activándose entonces la síntesis de ciertos aminoácidos. Se ha encontrado también que en las zonas altoandinas, en donde la proporción de radiación ultravioleta es mayor en relación a la costa, las 10 variedades de papa estudiadas presentan un mayor contenido proteico Calderón (1988).

Los contenidos de prolina indicaron una mayor concentración en las plantas crecidas en el medio IRRI+N que aquellas crecidas en el medio IRRI-N con valores de $1.87 \mathrm{mg} / \mathrm{g}$ y $0.97 \mathrm{mg} / \mathrm{g}$ respectivamente. Estos valores sugieren un cierto grado de estrés, que a su vez coinciden con las observaciones cualitativas y cuantitativas hechas en las plantas crecidas en el medio con $\mathrm{NO}_{3}{ }^{-} \mathrm{NH}_{4}$, los cuales tuvieron tasas bajas de crecimiento y debilidad de las frondas. La prolina en niveles altos es un indicador de stress (Bates, 1973).

Con relación a las actividades de las enzimas Nitrogenasa y Nitrato reductasa, no se mostraron diferencias significativas en los diversos tratamientos, sin embargo, se observó cierta tendencia hacía una mayor actividad Nitrogenasa en las plantas crecidas sin Nitrógeno, a su vez una mayor actividad Nitrato Reductasa en aquellas plantas crecidas con suplemento nitrogenado. Lo observado tiene concordancia con los resultados de Zavaleta (1993) el sentido de que el sustrato induce la síntesis y la actividad de la Nitrato Reductaza. y a la vez la carencia de Nitrógeno en el sustrato, capacita a los simbiontes a fijar la proporción de Nitrógeno para cubrir las necesidades. Por otro lado, dado que la luz azul y la luz roja tienen el mismo efecto en la fotosíntesis, entonces la provisión de energía para la fijación y de ferredoxina para la reducción tiende a ser la misma, quedando la pequeña diferencia solo debido 
al efecto del nitrógeno en el sustrato (La Rue \& Kuez,1973; Moses \& Chua, 1988).

\section{Conclusiones}

Las tasas de crecimiento relacionadas al peso fresco como al área foliar y la cantidad de células vegetativas y heterocistos mostraron valores promedio significativamente mayores en las plantas crecidas en el medio IRRI-N.

Los contenidos de clorofila a, b y totales, así como la prolina presentaron valores promedio significativamente mayores en las plantas crecidas en el medio IRRI $+\mathrm{N}$.

En cuanto al contenido de proteínas, las plantas crecidas bajo luz azul presentaron contenidos promedio significativamente mayores que aquellas crecidas bajo luz roja y blanca respectivamente.

Finalmente podemos decir que la ausencia de Nitrógeno en el medio del cultivo influye de modo significativo en el crecimiento de la planta y de su endosimbionte, aunque las plántulas se vean de un tono más amarillento (no clorótico) por la menor síntesis de clorofilas.

\section{Literatura citada}

Ali S. \& Malik K.A.1983. Use of Azolla in Pakistan. Nuclear Institute for Agriculture and Biology. Current Science (Faisalabad, Pakistan). :179-185.

Alves M. 1985. Azolla - Anabaena azollae. Ministerio de Educación - Faculdade de Ciencias Agrarias do Pará. Brasil.

Bates L. 1973. Rapid determination of free proline for water stress studies.Plant and Soil. 39: 205 - 207.

Becking J.H. 1978. Ecology and physiological adaptation of Anabaena in the Azolla - Anabaena azollae symbiosis. Ecological Bulletins (Granhall. Stockholm). 26: 266 - 281

Calderón R.A. 1988. Efectos de dos microclimas contrastantes en la composición Bioquímica de los tubérculos de diez genotipos de papa. Tesis para optar el título de Biólogo. Universidad Nacional Agraria La Molina. Lima - Perú.

Drevon J. 1993. Evaluación de la actividad nitrogenásica de los nódulos de las leguminosas mediante la actividad reductora del acetileno. FAO/ GRET. I- BIOL 5 (Roma).: $1-5$.

Fay P. 1973. The Heterocysts: The Biology of Blue Green Algae. Carre and Whitton (edts). University of California. : 239 - 259

Fay P. 1992. Oxygen Relations of Nitrogen Fixation in Cyanobacteria. Microbiological Reviews. 56 (2): $340-373$.
Franco A.A., Pereira J.C. \& Neyra C.A. 1979. Seasonal patterns of nitrate reductase and nitrogenase activities in Phaseolus vulgaris $\mathrm{L}$. Plant Physiology. 63: 421 - 424.

Grossman A.R., Schaefer M.R., Chiang G.G. \& Collier J.L. 1993. The Phycobilisome, a Light Harvesting Complex Responsive to Environmental Condition. Microbiological Reviews. 157 (3): 725 -749 .

Gornall A.G., Bardawill C.S. \& David M. 1949. Determination of Proteins by Biuret. Journal. Biol. Chem 1.77: 751 - 753 .

Katiyar V. 1996. Determination of Proteins by the Technique of Biuret. Biochemistry Laboratory Manual IAU. Dept. of Chemistry.

La Rue T.A. \& Kuez W.G. 1973. Estimation of nitrogenase using a colorimetric determination for ethylene. Plant Physiology. 51: $1074-1075$.

Moses P.B. \& Chua N.H. 1988. Light switches for plant genes. Scientific American 258 (4): 88 - 93.

Neidhardt C.F., Ingraham J.L., y Schaechter M. 1990. Physiology of the Bacterial Cell. Sinauer Associates. I.N.C. (Massachusetts E.U.). : 448 451.

Paniagua J. y Sasson A. 1995. Moléculas de Microalgas de Importancia Económica. Manual de Métodos Ficológicos. Universidad de Concepción - Chile.

Peters G.A. \& Calvert H.E. 1983. The azolla Anabaena azollae Simbiosis. In Algal Simbiosis (New York, Cambridge): $109-145$. University Press.

Reddy P.M. \& Fisher R.W. 1988. A nobel method to produce Anabaena - free Azolla by in vitro fertilization of micromanipulated megasporocarps. Plant Cell Reports. 7: 430 - 433.

Tovar D.T. 1993. Capacidad de Fijación de Nitrógeno de los Ficobiontes aislados de líquenes del género Leptogium S.Gray. Revista de Química (Lima Perú). 7(2): $137-154$.

Yatazawa M., Tomomatzu N., Hosoda N. \& Nunone K. 1980. Nitrogen Fixation in Azolla - Anabaena simbiosis as affected by mineral nutrient status. Soil Science and Plant Nutrition 26: 415 - 426.

Zavaleta A.J. 1992. Evaluación del efecto de la fertilización nitrogenada sobre la actividad de la Nitrogenasa y la Nitrato Reductasa en Phaseolus vulgaris L.var. canario corriente. Tesis para optar el título de Ingeniero Agrónomo Universidad Nacional Agraria La Molina Lima Perú. 


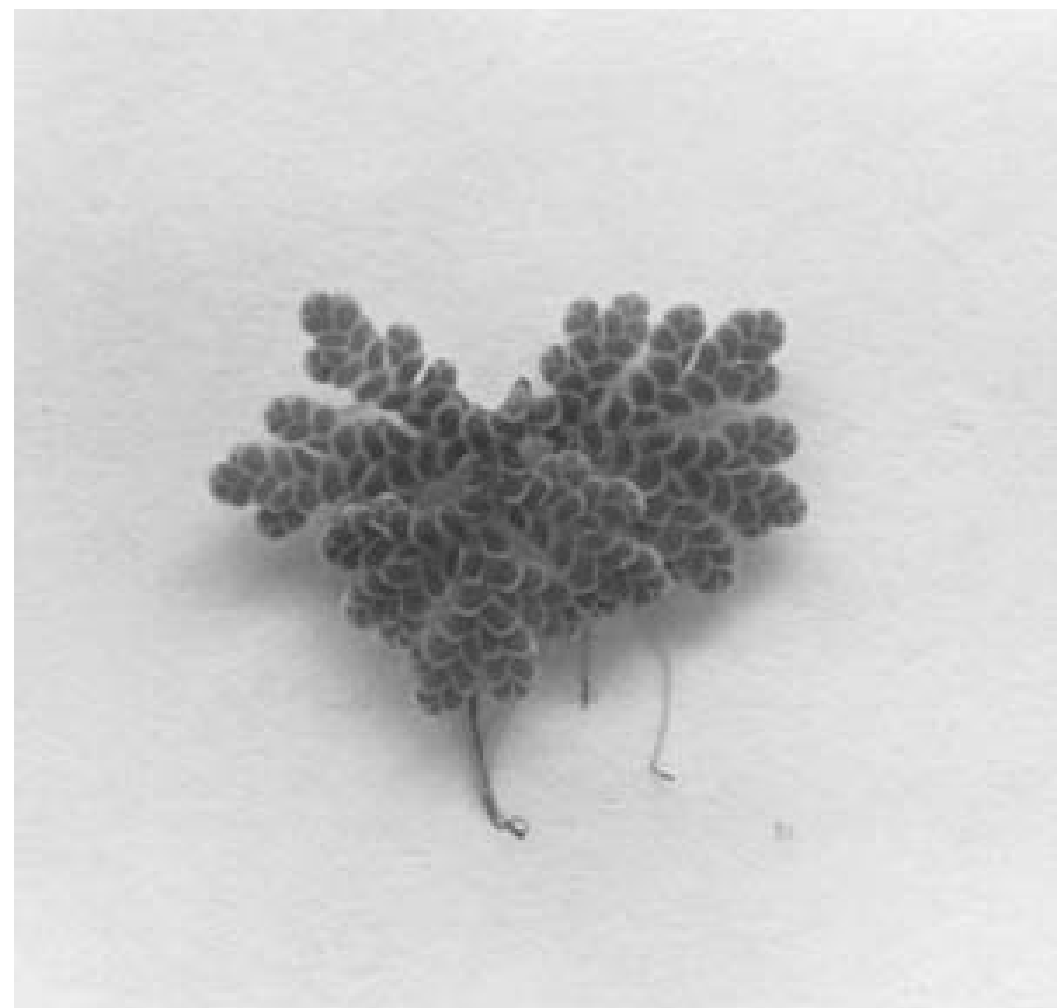

Figura 1. Azolla filiculoides Lam, pequeño helecho acuático flotante que vive en muchos espejos de agua dulce y en áreas tropicales y es capaz de fijar nitrógeno atmosférico debido a la simbiosis que mantiene con la cianobacteria Anabaena azollae Stras.

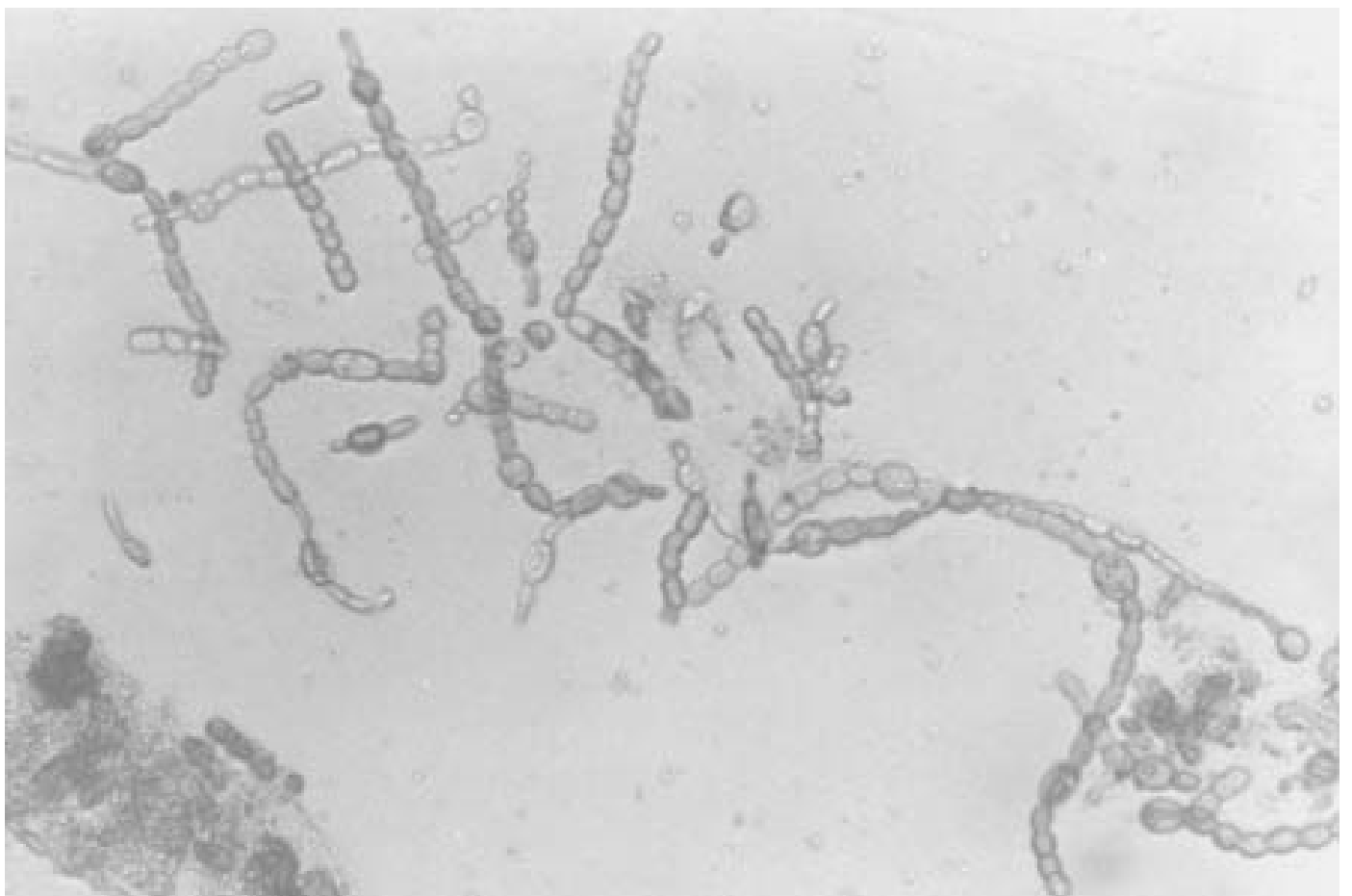

Figura 2. Anabaena azollae Stras, habita en unas grandes cavidades en el lóbulo superior de la fronda de Azolla. 


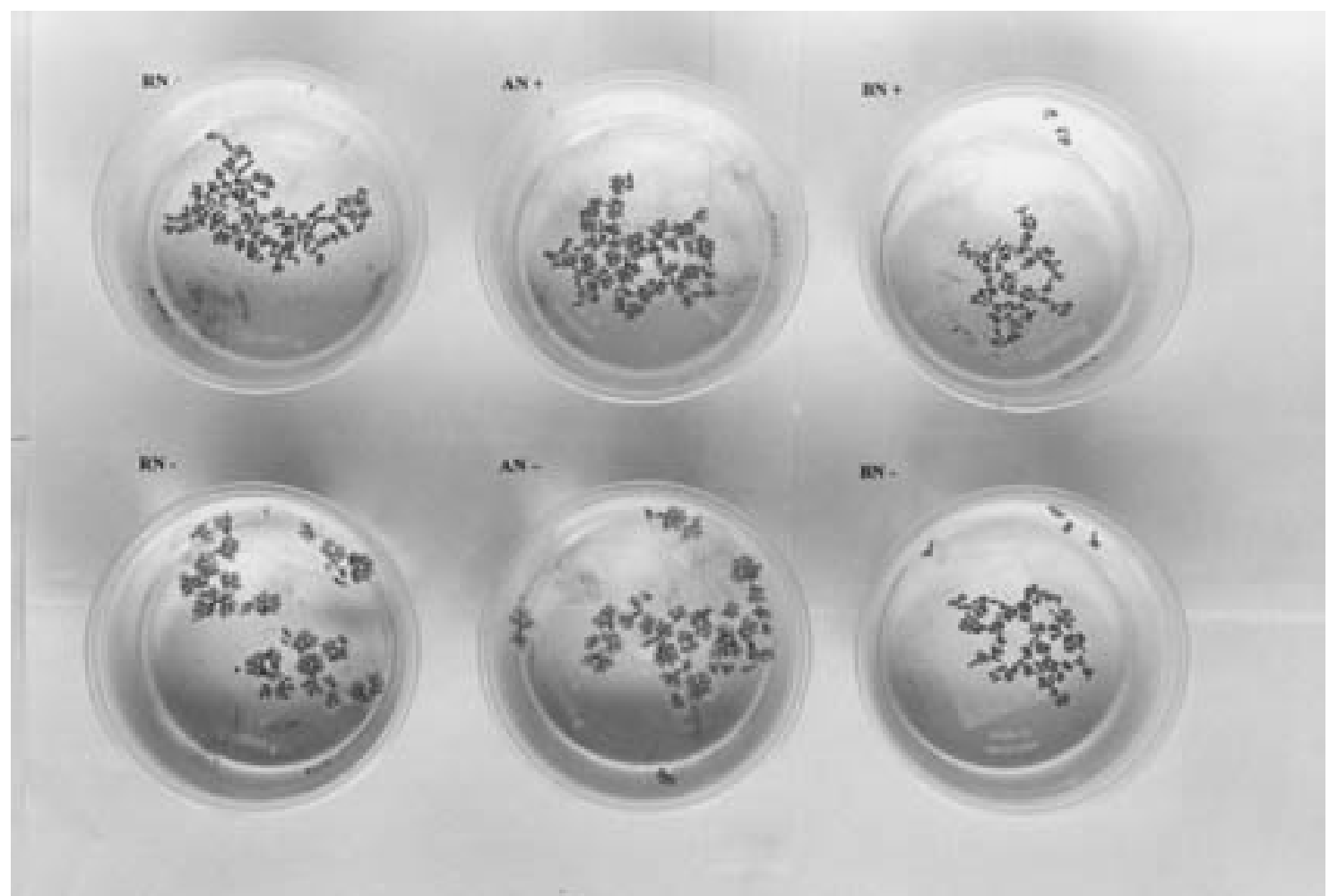

Figura 3. Contenedores plásticos de $500 \mathrm{ml}$, cada nivel de nitrógeno fue colocado bajo las diferentes calidades de luz (roja, azul y blanca). El fotoperíodo fue de 12 horas de luz y 12 de oscuridad.

Tabla 2. Parámetros bioquímicos evaluados.

\begin{tabular}{|l|c|c|}
\hline \multicolumn{1}{|c|}{ LUZ AZUL, LUZ ROJA, LUZ BLANCA. } & IRRI -N & IRRI + N \\
\hline Tasa de crecimiento por día & 0.092 & 0.027 \\
Cantidad total de heterocistos y células vegetativas & 29.16 & 10.44 \\
(promedio) & 0.049 & 0.085 \\
Clorofilas a, b y totales (promedio) & 0.97 & 1.87 \\
Contenido de prolina $\mathrm{mg} / \mathrm{g}$ & 0.174 & 0.273 \\
Nitrato reductasa. $\mu$ moles $\mathrm{NO}_{3}{ }^{-} / \mathrm{hora} / \mathrm{g}$ PF & 26.765 & 35.348 \\
Nitrogenasa. $\mu$ moles acetileno reducido/g PF/hora & \multicolumn{2}{|c|}{45.47} \\
\hline Proteína $\mathrm{mg} / \mathrm{g}$ & Luz azul & 26.08 \\
\hline
\end{tabular}

